\title{
On the Chronobiology of Cohabitation
}

\author{
M.J. PAUL AND W.J. SChWARTZ \\ Department of Neurology, University of Massachusetts Medical School,
} Worcester, Massachusetts 01655

\begin{abstract}
Social regulation of animal circadian rhythms may enable individuals in a population to temporally synchronize or segregate their activities within the community. Relatively little is known about the mechanisms for such interindividual temporal adaptations or how the circadian system might be involved. The literature suggests that actual prolonged cohabitation might lead to robust effects on the rhythmicity of cohoused individuals but that these effects are not easily reproduced by indirect or pulsatile social contacts. We have begun to study the conditions under which such cohabitation effects might be revealed in the laboratory, and we present and discuss initial data that cohousing pairs of golden hamsters can result in a persistent change in the free-running circadian period of one of the two hamsters of the pair. We believe that analyzing the societal level of temporal organization, and ultimately dissecting its underlying mechanisms, will enrich our understanding of the circadian clock and its role in establishing ecological communities.
\end{abstract}

\section{INTRODUCTION}

Our knowledge of the circadian system of animals at the molecular, cellular, tissue, and organismal levels is remarkable, and we are beginning to understand how each of these levels contributes to the emergent properties and increased complexity of the system as a whole. For the most part, analysis has been performed using singly housed animals in plastic cages with temperature, humidity, and access to food rigidly controlled. Of course, many species ordinarily would not live out their lives in such seclusion. Some live in colonies with highly developed social structures and a clear division of labor, requiring modifications to daily rhythms; in bees, for example, foragers periodically leave the hive and express robust circadian activity rhythms, whereas nurses care for the brood continuously and are active during both the day and the night (Shemesh et al. 2007). Social mediation of honeybee (Apis mellifera) temporal organization becomes apparent when the colony experiences a shortage of nurses. Foragers return to nursing and adopt an arrhythmic, persistently active state (Bloch and Robinson 2001). In the wild, animals living together might synchronize their behaviors to achieve common goals or, alternatively, actively avoid each other to lessen competition for limited resources. At this supra-organismal level of organization, how are such interindividual daily adaptations achieved in order to form real ecological communities? Is the circadian system involved, and what mechanisms are responsible?

\section{SYNCHRONIZATION AND SEGREGATION OF ANIMALS IN THE FIELD AND THE LABORATORY}

Two recent reviews (Davidson and Menaker 2003; Mistlberger and Skene 2004) summarize our knowledge of social cues and the expression of circadian rhythmicity, highlighting the conceptual, experimental, and speciesspecific complications of this research. In the field, temporal synchronization among animals has been inferred from studies of Canadian beavers (Castor canadensis) living together as a family unit, as they exhibit a single coherent free-running rhythm in daily noise while they overwinter in constant darkness (DD) under thick ice (Bovet and Oertli 1974; Potvin and Bovet 1975). The period $(\tau)$ of the free-running rhythm does not correspond to any environmental factor, suggesting that social interactions alone are responsible for the synchronous group activity. In the laboratory, several studies have demonstrated that cohoused or group-housed animals - including killifish (Fundulus heteroclitus; Kavaliers 1980), deer mice (Peromyscus maniculatus; Crowley and Bovet 1980), palm squirrels (Funambulus pennanti; Rajaratnam and Redman 1999), and fruit flies (Drosophila melanogaster; Levine et al. 2002) — may display coherent or converging activity rhythms, perhaps of altered amplitude or waveform, under constant lighting conditions (for reviews, see Regal and Connolly 1980; Davidson and Menaker 2003; Mistlberger and Skene 2004). Although these studies have established that cohabitation can alter the daily activity pattern in several species, their reliance on group activity data has prohibited any further chronobiological or mechanistic analyses. The expression of a single free-running rhythm in group-housed individuals could be due to social entrainment (mutual or unidirectional), but it could also reflect similar phases and $\tau$ values before cohabitation or a masking effect of one, possibly socially dominant, individual. To distinguish between these alternatives, the activity of each individual must be identified before, during, and after cohabitation.

Various studies have used different strategies to distinguish individual activity under social conditions. First, investigators have housed animals in close proximity to, but not in direct contact with, each other. Under these housing conditions, social effects on the circadian system depend on the species but appear to be quite modest. When animals are housed in the same cage but separated by a barrier (e.g., wire mesh, clear perforated plastic, or a restraining cage), studies have reported alterations in activity levels, reentrainment rates to a photically induced 
phase-shift, or $\tau$ values in DD (e.g., in Octodon degus; Goel and Lee 1995, 1996, 1997), but clear examples of temporal synchronization have not been obtained. One study has demonstrated synchronous activity due to social cues in individuals separated by a barrier. Caged microchiropteran bats (Hipposideros speoris) housed in a cave without access to light synchronize their activity rhythms to local time, in-phase with their free-living conspecifics that leave the nest daily around sunset; a control bat caged in a cave devoid of other bats instead exhibited a free-running rhythm (Marimuthu et al. 1981).

Some studies have shown circadian effects in some individuals upon presentation of a putative social cue alone. Supplying female degus with an odor cue from entrained degus has been reported to "temporarily" entrain some of the animals and to accelerate reentrainment to a phase-advanced light/dark cycle (Governale and Lee 2001; Jechura et al. 2006). Some bird species can entrain to playbacks of conspecific birdsong presented every 24 hours (Gwinner 1966; Menaker and Eskin 1966), but house sparrows (Passer domesticus) can also entrain to periodic noise (Reebs 1989), so it is not clear if entrainment to birdsong playback is truly a social phenomenon or the result of nonspecific arousal.

Some animals may segregate, rather than synchronize, their activity times, perhaps to avoid competition for a particular temporal niche. For example, two species of closely related spiny mice (Acomys cahirinus and Acomys russatus) are able to cohabit in the same rocky terrain of the Dead Sea Valley by assuming different activity phases: $A$. cahirinus is nocturnal and $A$. russatus is diurnal (Shkolnick 1971). When $A$. cahirinus is removed from the field site, $A$. russatus becomes nocturnal, indicating that the presence of A. cahirinus prevents $A$. russatus from being active at its preferred phase of the daily cycle. When $A$. cahirinus is released back into the field site, both species reestablish their temporal segregation. Importantly, light does not regulate this phenomenon. Similar examples of competitively displaced temporal activity patterns have been described between and across other species under seminatural conditions (Glass and Slade 1980; Ziv et al. 1993). In the laboratory, A. cahirinus and A. russatus are strictly and primarily nocturnal, respectively (Weber and Hohn 2005; Cohen and Kronfeld-Schor 2006), and chemical signals from A. cahirinus have been shown to alter the phase angle of entrainment of $A$. russatus, suggesting that these cues contribute to their temporal partitioning reported in the field (Haim and Rozenfeld 1993; Fluxman and Haim 1993). There is also a report of laboratory group housing of long-tailed field mice (Apodemus sylvaticus), in which three subordinate mice were observed to avoid the time of peak out-of-burrow activity of a dominant mouse (Bovet 1972).

\section{SEARCHING FOR TEMPORAL COUPLING BETWEEN GOLDEN HAMSTERS}

Golden hamsters (Mesocricetus auratus) would seem to be well-suited as a laboratory species for investigating the circadian effects of cohabitation. They are widely available, exhibit precise locomotor activity (wheel-running) rhythms with sharp activity onsets, respond predictably to a range of nonphotic phase-shifting cues (Mrosovsky 1996), and have been experimental subjects for dissecting the neurobiological and hormonal substrates that underlie social interactions, especially agonistic (conflict) behaviors (Albers et al. 2002; Huhman 2006).

There is an inconsistent literature thus far on social cues and circadian rhythm resetting in hamsters. Closely housed or cohoused hamsters not in physical contact do not affect each other's rhythms (Davis et al. 1987; Gattermann and Weinandy 1997), although more rapid reentrainment to a phase-advanced light/dark cycle has been reported when males are paired with estrous females (Honrado and Mrosovsky 1989). Other studies have restricted the interval of social contact to a few hours per day, ensuring that the activity during the remaining hours is due solely to the experimental animal. This approach has led to conflicting results (Aschoff and von Goetz 1988; Mrosovsky 1988; Refinetti et al. 1992; Mistlberger et al. 2003), perhaps due to differences in experimental design (blind vs. intact animals, DD vs. constant light, interaction via neutral cage vs. resident-intruder paradigm). Refinetti et al. (1992) cohoused two hamsters in physical contact with each other but with markedly different $\tau$ values: One was entrained to a short 23.3-hour $\mathrm{T}$ cycle, whereas the other was blinded and free-ran with a $\tau$ value of about 24 hours. There was no synchronization or relative coordination of the activity of the blind hamster to that of the entrained one. The conclusion from all these studies has been that social factors provide only weak inputs to the hamster circadian clock (Davidson and Menaker 2003; Mistlberger and Skene 2004).

Even though social interactions may be weak, their effects on behavior might be strong under certain circumstances. If we consider hamsters as individual oscillators, and their social interactions as a form of weak coupling, then one would predict that strong effects might be precipitated when cohabitants are in direct physical contact for a relatively long period of time and their respective $\tau$ values are close to one another (Winfree 2001). To our knowledge, no reported experiment in hamsters has incorporated such a design, which should be more sensitive for inducing and detecting the possible effects of social interactions than the studies previously described. We have now performed such an experiment and report our initial observations here.

\section{COHOUSING HAMSTERS LEADS TO A PERSISTENT $\tau$ CHANGE IN ONE HAMSTER OF A PAIR}

Baseline wheel-running activity was recorded in adult male golden hamsters singly housed in DD for 3-7 weeks. Sixteen hamsters with dissimilar $\tau$ values were selected to yield eight cohabiting pairs. The mean $( \pm$ S.E.M. $) \tau$ for the population as a whole was $24.12 \pm 0.03$ hours (range 23.95-24.32 hours), and the mean difference in $\tau$ between the two hamsters of each pair before cohousing was 0.18 \pm 0.04 hours. To decrease the incidence of fighting at the time of pairing, hamsters were cohoused in a fresh cage with nesting material available. A cage lid equipped with a single running wheel was positioned on top of the cage, 


\section{CHRONOBIOLOGY OF COHABITATION}

and behavioral observations were recorded for 30 minutes under dim red light at the time of pairing, with specific attention given to aggressive behaviors and postures. During cohabitation, hamsters were checked regularly for behavioral interactions and signs of physical discomfort (initially once a day during the first week, then about 3 times per week thereafter).

The number of wheel revolutions for each hamster or hamster pair was binned every 15 minutes and plotted at 24-hour intervals with successive days stacked vertically to form single-plotted actograms. $\tau$ was determined by drawing an eye-fitted regression line over 7-10 successive activity onsets immediately before cohabitation and after separation using the $\tau$ cursor function in the Actiview program (Mini-Mitter Co., Inc., Oregon). Cycle-to-cycle variability was determined by first computing the periods of ten successive cycles using the same $\tau$ cursor function; onsets occurring after cage changing were excluded from analyses. The standard deviation of these ten cycle lengths was then calculated to provide a measure of $\tau$ variability for each hamster. Mean daily activity was calculated by averaging the number of wheel revolutions occurring in a 24-hour interval over 10 successive days.

Wheel-running activities before pairing, during cohabitation, and after separation for two pairs of hamsters are illustrated in Figure 1. In both cases, an obvious and sudden change in $\tau$ of one of the cohabitants was observed. Hamster activities appeared to temporally "collide" when the locomotor offsets of nos. 7 and 34 intersected the locomotor onsets of nos. 8 and 20, respectively. The effect was a clear shortening of $\tau$ in nos. 7 and $34(-0.21$ and -0.19 hour, respectively), resulting in a temporal separation of the hamsters' wheel running in their cages in $\mathrm{DD}$; the $\tau$ change persisted after the hamsters were separated and singly housed.

Figure 2 shows the $\tau$ values for hamsters of all eight pairs before cohabitation and after separation. The $\tau$ change associated with cohabitation was generally asymmetric, with one hamster of the pair exhibiting a larger change in $\tau$ than that of its cohabitant; and this new $\tau$ continued to be expressed by the affected hamster after the pair was separated (and stably maintained in the cases we followed for weeks to months). The mean $\tau$ change in relatively "affected" versus "unaffected" hamsters of the pairs was $0.19 \pm 0.02$ hour versus $0.05 \pm 0.01$ hour, respectively, and the difference between these two populations was significant (Student's $t$-test, $p<0.05$ ). Social rank, as assessed by dominance status at pairing and again just before separation, could not be confidently determined for three of the pairs; for the remaining five pairs, the affected hamster was dominant in three and subordinate in two.

It is interesting to note that in seven of our eight pairs, the affected hamster's $\tau$ was shortened by cohabitation; in these cases, the affected hamster was the animal that had exhibited the longer $\tau$ of the pair before cohabitation. There was a trend toward increased cycle-to-cycle variability (i.e., decreased precision of the locomotor rhythm) in the precohabitation activity of the hamster of each pair whose $\tau$ would later be shortened by cohabitation ( $\tau$ variability before cohabitation, $0.45 \pm 0.09$ hour in hamsters
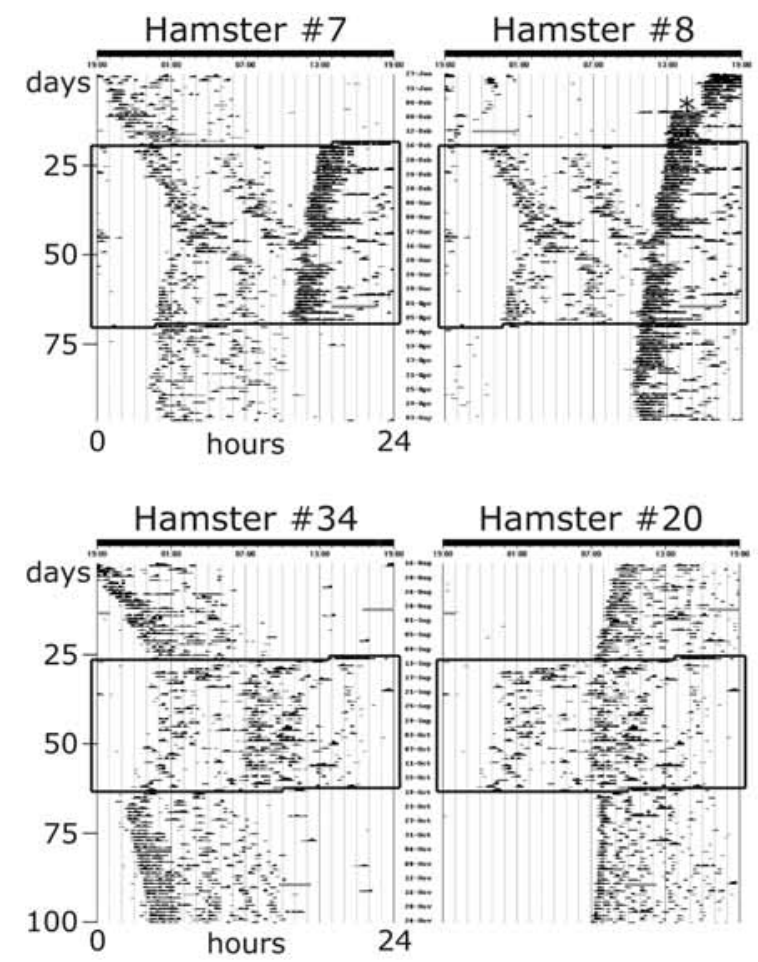

Figure 1. Wheel-running activity in DD of pairs of hamsters (top, no. 7 with no. 8; bottom, no. 34 with no. 20) before, during, and after cohabitation. Activity is illustrated as single-plotted actograms with consecutive 24-hour intervals drawn on successive lines. Activity enclosed within the boxes represents wheelrunning behavior during cohabitation; note that this activity is identical for the hamsters within each pair and that individual activity cannot be conclusively determined. Asterisk $(*)$ marks a large phase-shift associated with an anesthetization procedure on hamster no. 8 before cohabitation. (Gray bars) Intervals of lost computer function.

whose $\tau$ was subsequently "affected" by cohabitation vs. $0.24 \pm 0.05$ hour in hamsters whose $\tau$ was subsequently "unaffected" by cohabitation; $p=0.051$, Student's $t$-test), and a positive correlation between the degree of this imprecision and the magnitude of $\tau$ shortening $\left(r^{2}=0.29\right.$; $p=0.03)$. There was no difference in precision after separation ( $\tau$ variability after cohabitation, $0.24 \pm 0.05$ hour in hamsters whose $\tau$ was previously "affected" by cohab-

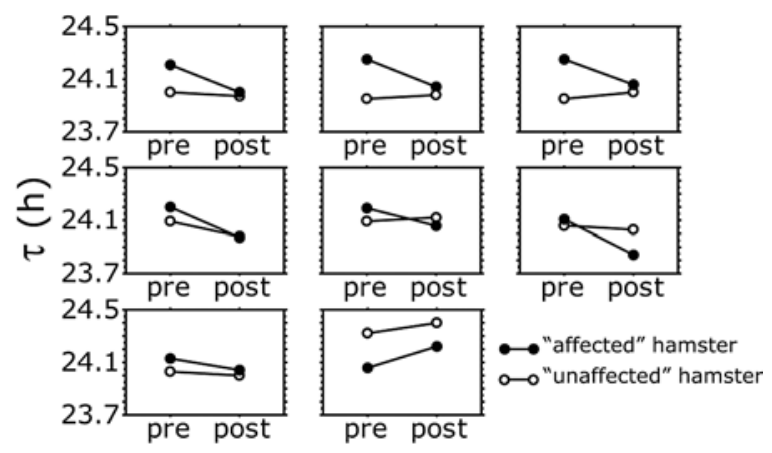

Figure 2. The value of $\tau$ before (pre) and after (post) cohabitation is shown in each box for the two hamsters of a pair, for a total of eight pairs. 
itation vs. $0.25 \pm 0.05$ hour in hamsters whose $\tau$ was previously "unaffected" by cohabitation). Mean daily activity counts did not differ between affected and unaffected hamsters either before cohabitation or after separation (4513 \pm 823 vs. $5059 \pm 978$ before and $3817 \pm 507$ vs. $3738 \pm 613$ after cohabitation, respectively, affected vs. unaffected hamsters; $p>0.05$ for each comparison, Student's $t$-test).

We also have examined a few additional cases in which hamsters with similar $\tau$ values were paired at the same phase; under these circumstances, it is obvious that individual activity rhythms could not be discriminated during cohabitation (Fig. 3). For one such pair (Fig. 3, top, note that both cohabitants in this instance were females), no circadian effects were apparent, whereas for another pair (Fig. 3, bottom) the result was a gross, persistent shortening of $\tau$ in hamster no. 65 . The hamsters of this particular pair were observed to be fighting 50 days after pairing, but they likely began fighting earlier than that because these hamsters, which initially shared the same nest, were seen on several occasions using separate nests beginning 28 days after pairing. Affected hamster no. 65 was the behaviorally dominant animal of this pair.

One other case of an initial pairing of hamsters with similar $\tau$ values at the same phase is shown in Figure 4. The $\tau$ of hamster no. 16 gradually shortened soon after pairing, after which the combined locomotor activities of hamsters nos. 16 and 5 were recorded for nearly 9 months. Visual inspection of the actogram shows that hamster no. 16 continued to be the affected hamster of the pair; the record hints that collisions of locomotor onsets and off-
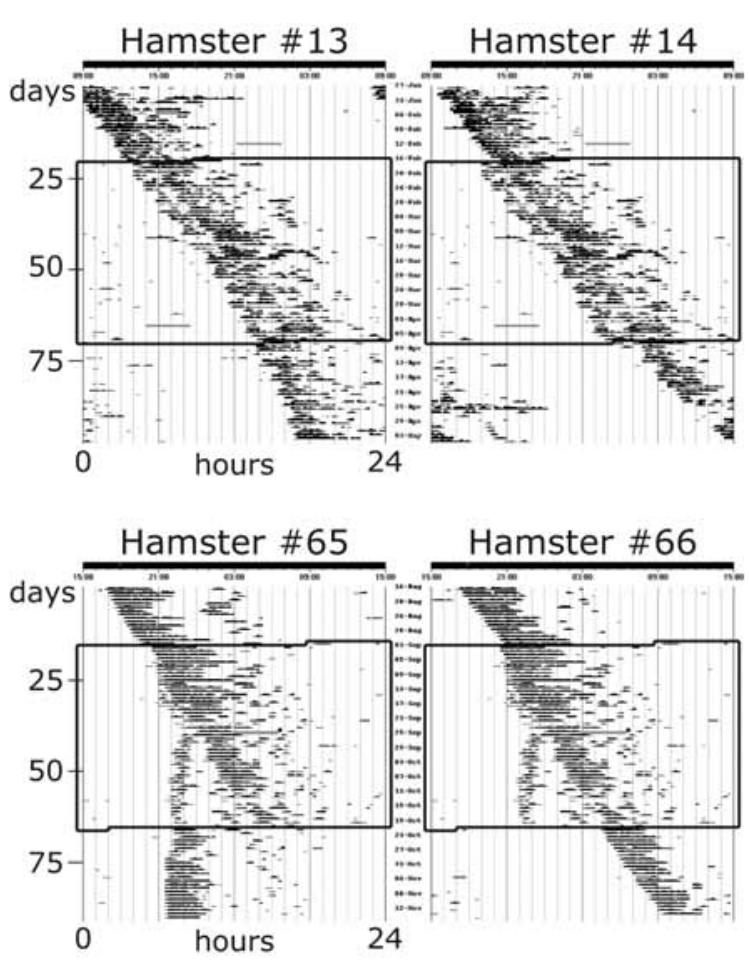

Figure 3. Wheel-running activity in DD of pairs of hamsters (top, no. 13 with no. 14; bottom, no. 65 with no. 66) before, during, and after cohabitation, illustrated as single-plotted actograms as in Fig. 1. sets might be a critical factor for inducing the change in $\tau$, although in this case its direction was unpredictablefirst shortened, then lengthened, and finally with no change at all (arrows, Fig. 4).

\section{TOWARD A CHRONOBIOLOGY OF COHABITATION}

Circadian rhythms are believed to be adaptive responses to the challenges of living on a rotating planet. The

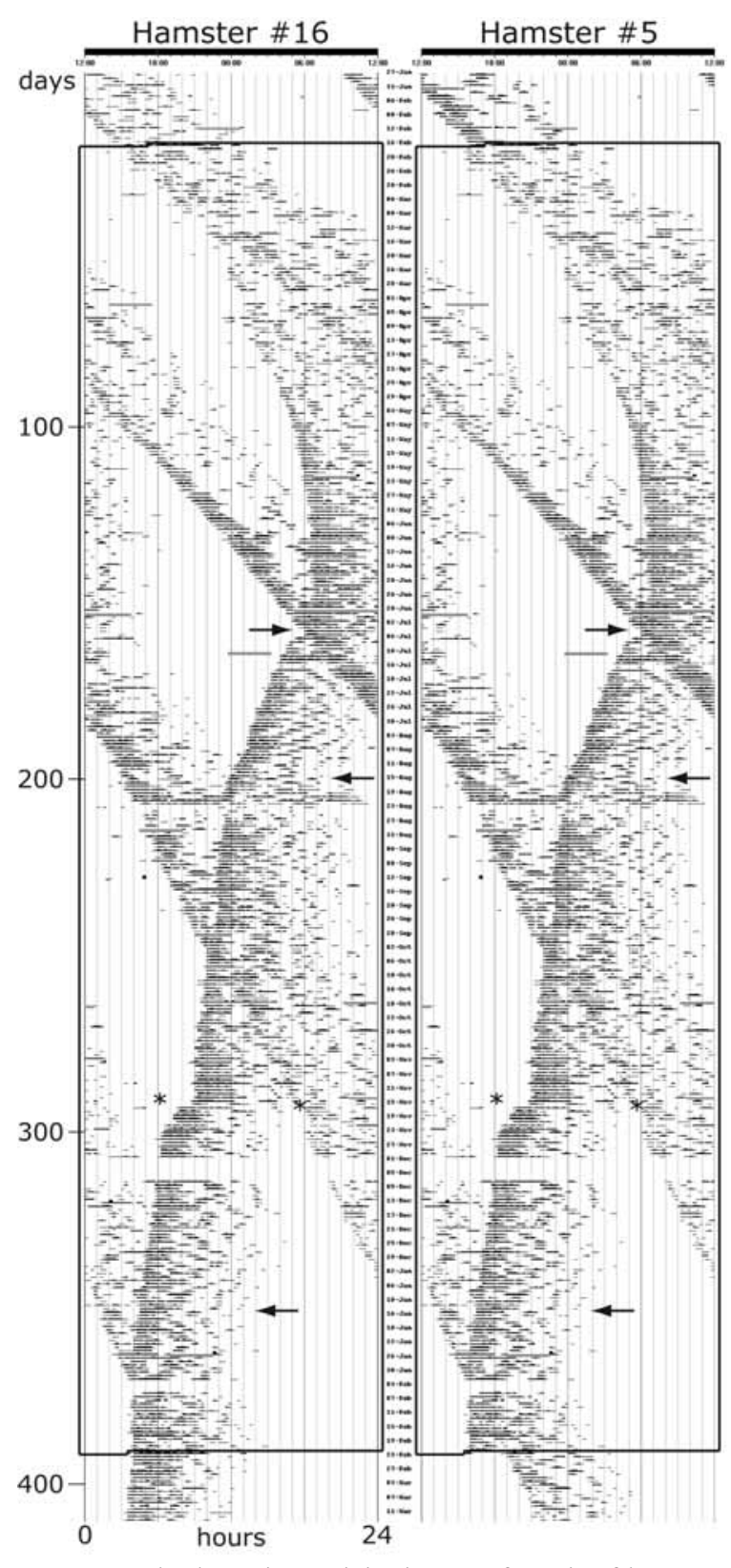

Figure 4. Wheel-running activity in DD of a pair of hamsters (no. 16 with no. 5) before, during, and after cohabitation, illustrated as single-plotted actograms as in Fig. 1. Arrows mark collisions of locomotor onsets and offsets between the hamsters. Asterisks (*) mark a phase-shift associated with food competition tests for dominance status. 


\section{CHRONOBIOLOGY OF COHABITATION}

generation of rhythmicity and its entrainment to the light/dark cycle have been the focus of much research, as represented by the reports in this volume, as well as by the work of this laboratory (W.J.S.) for the past few decades, from molecular to organismal levels. Relatively less attention has been directed to investigating the generation and entrainment of rhythmicity at the community level. The importance of social cues may be as a mechanism for adjusting the phase of individuals within a population to a shared photic environment. We have observed a persistent, cohabitation-associated, statistically significant shortening of $\tau$ in DD that might be functionally significant. If such a change were stable and affected individuals differentially, it could translate to an altered phase angle of entrainment to a light/dark cycle, with temporal partitioning of activity between cohabiting animals as a result.

One potential confound of our experiment is that the change in $\tau$ might reflect a seasonal change of behavior or physiology that accompanies the transfer of hamsters to short-day or DD conditions, rather than to cohabitation per se. For example, the effect might be secondary to the decreased testicular hormone secretion characteristic of the short-day condition. We believe that this is unlikely, however; castration lengthens, rather than shortens, $\tau$ in mice (Daan et al. 1975) and in fact does not seem to affect the value of $\tau$ in golden hamsters (Morin and Cummings 1981). Elliott and Tamarkin (1994) have shown no change in $\tau$ of hamsters maintained in DD for varying lengths of time ( 5 days, 7 weeks, 12 weeks, and 27 weeks).

It is noteworthy that the $\tau$ change predominantly affects one hamster of a pair and is sustained (for months in the few hamsters that we have followed long-term). Such a persistent behavioral change in response to a social encounter reminds us of the phenomenon of "conditioned defeat" in golden hamsters (for review, see Huhman 2006). After a hamster has been defeated in a single encounter with a more aggressive male, it remains submissive and defensive when tested by further agonistic encounters with smaller, nonaggressive opponents ("loser effect"), an outcome that can last for at least 1 month. We emphasize that in our experiments, the level of aggression generally appears to be modest, not leading to physical injury, and cage mates often huddle together in the same nest. It seems unlikely that our cohabitation effects are due to continuous or extreme arousal, and indeed, social defeat stress is not believed to perturb the period, phase, or amplitude of the central circadian pacemaker that regulates behavioral rhythms (Meerlo et al. 2002).

We do not understand why cohousing is associated with a preferential change in one of the two cohabitants' $\tau$, although the affected individual's longer $\tau$ and less precise rhythm before cohabitation may be a clue. Our next step must include the capability to distinguish the activity of individual animals housed together, and implantable miniature temperature sensors are already commercially available for this purpose. We have also custom designed a "micrologger" that measures general locomotor activity and is small enough to implant in hamsters (manufactured by Sigma Delta Technologies, Perth, Australia). The logger senses movement using an accelerometer, collecting at two samples/second and integrating over 5 minutes using a microprocessor equipped with a $4-\mathrm{MHz}$ clock. The 5-minute integration is then stored in a memory chip. The logger is powered by an attached battery and thus functions as a self-contained activity acquisition and storage unit enabling measurement in individual animals for up to 6 months, without interference from transmissions generated by nearby devices. The board is $1.5 \times 1.7 \mathrm{~cm}$ and weighs approximately 3 grams; a Teflon tape wrap and custom casing fabricated from acrylonitrile butadiene styrene protect the device from damaging water vapor. The implanted device does not inhibit hamster wheel running (Fig. 5), encouraging us to begin to ask questions about individual hamster activities during cohabitation (e.g., is the change in $\tau$ dependent on the phase relationship of the cohabitants' rhythms, especially at the apparent temporal "collisions" seen in Figs. 1 and 4?). We also should be able to analyze the influence of the running wheel itself; we already know that several nonphotic zeitgebers are thought to rely on at least some level of locomotor activity (Mistlberger et al. 2003), and the effects of nonphotic zeitgebers are often correlated with the amount of wheel-running behavior these manipulations elicit (for review, see Mrosovsky 1996).

Ultimately, we would hope to implicate specific neurobiological circuits as mediators of our effects and at least one candidate system immediately comes to mind. In rodents, aggression, affiliative behavior, and social recognition are regulated, in part, by the peptide neurotransmitter vasopressin (for review, see Albers et al. 2002), and vasopressin $\mathrm{V}_{1 \mathrm{a}}$ receptor mRNA and radioligand binding have been mapped in the golden hamster and are abundantly present in the suprachiasmatic nucleus (Young et al. 2000), site of the central circadian pacemaker regulating locomotor rhythmicity.

\section{CONCLUSIONS}

As we begin this line of research, we have many more questions than conclusions. We do know that our expertise in circadian biology needs to be supplemented by

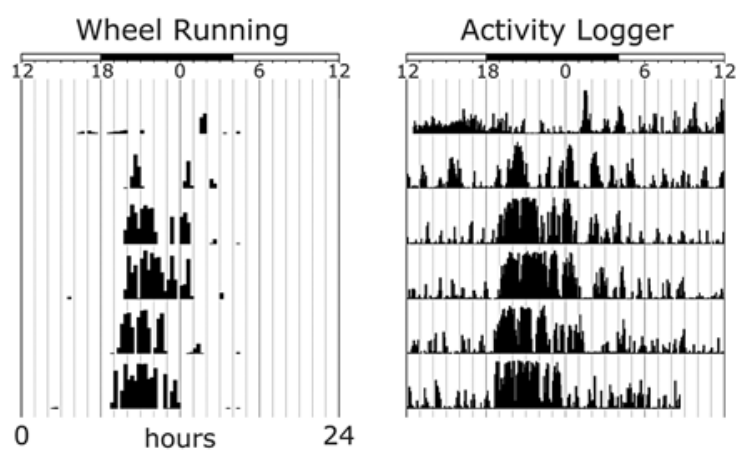

Figure 5. Locomotor activity of a single golden hamster as recorded by a running wheel (left) or subcutaneously implanted micrologger (right). Activity is illustrated as a single-plotted actogram. The hamster was maintained in a 14-10-hour light/dark cycle as indicated by the white and black bars at the top of the actogram. As expected, wheel running accounts for only a part of the animal's total 24-hour activity. 
more knowledge of behavioral ecology and social biology. We also appreciate that our $\tau$ findings in hamsters may reflect an unnatural laboratory situation (e.g., being trapped in a relatively small enclosure with a cage mate) and might not be relevant for this species in its natural habitat. Right now, we are at the beginning, gathering data to rekindle interest in this problem, to encourage future experiments under more naturalistic conditions, and eventually to test hypotheses in field studies. We submit that analyzing this interorganismal and supraorganismal dimension of temporal organization, and ultimately dissecting its underlying mechanisms, will enrich our understanding of the circadian clock and the chronobiology of societies, perhaps providing insights into natural function, but certainly providing data on the social interactions of laboratory rodents that are routinely cohoused every day in our own biomedical facilities.

\section{ACKNOWLEDGMENTS}

We thank Dr. David Paydarfar for helpful discussions. This work was supported by NINDS F32 NS058135 (M.J.P.), and R01 NS46605 (W.J.S.). The contents of this report are solely the responsibility of the authors and do not necessarily represent the official views of the National Institutes of Health.

\section{REFERENCES}

Albers H.E., Huhman K.L., and Meisl R.L. 2002. Hormonal basis of social conflict and communication. In Hormones, brain and behavior (ed. D.W. Pfaff et al.), vol. 1, p. 393. Academic Press, San Diego, California.

Aschoff J. and von Goetz C. 1988. Masking of circadian activity rhythms in male golden hamsters by the presence of females. Behav. Ecol. Sociobiol. 22: 409.

Bloch G. and Robinson G.E. 2001. Reversal of honeybee behavioural rhythms. Nature 410: 1040.

Bovet J. 1972. On the social behavior in a stable group of longtailed field mice (Apodemus sylvaticus). II. Its relations with distribution of daily activity. Behaviour 41: 55.

Bovet J. and Oertli E.F. 1974. Free-running circadian activity rhythms in free living beaver (Castor canadensis). J. Comp. Physiol. 92: 1 .

Cohen R. and Kronfeld-Schor N. 2006. Individual variability and photic entrainment of circadian rhythms in golden spiny mice. Physiol. Behav. 87: 563.

Crowley M. and Bovet J. 1980. Social synchronization of circadian rhythms in deer mice (Peromyscus maniculatus). Behav. Ecol. Sociobiol. 7: 99.

Daan S., Damassa D., Pittendrigh C.S., and Smith E.R. 1975. An effect of castration and testosterone replacement on a circadian pacemaker in mice (Mus musculus). Proc. Natl. Acad. Sci. 72: 3744 .

Davidson A.J. and Menaker M. 2003. Birds of a feather clock together-Sometimes: Social synchronization of circadian rhythms. Curr. Opin. Neurobiol. 13: 765.

Davis F.C., Stice S., and Menaker M. 1987. Activity and reproductive state in the hamster: Independent control by social stimuli and a circadian pacemaker. Physiol. Behav. 40: 583.

Elliott J.A. and Tamarkin L. 1994. Complex circadian regulation of pineal melatonin and wheel-running in Syrian hamsters. $J$. Comp. Physiol. A 174: 469.

Fluxman S. and Haim A. 1993. Daily rhythms of body temperature in Acomys russatus: The response to chemical signals released by Acomys cahirinus. Chronobiol. Int. 10: 159.

Gattermann R. and Weinandy R. 1997. Lack of social entrainment of circadian activity rhythms in the solitary golden ham- ster and in the highly social Mongolian gerbil. Biol. Rhythm Res. 28: 85.

Glass G.E. and Slade N.A. 1980. The effect of Sigmodon hispidus on spatial and temporal activity of Microtus ochrogaster: Evidence for competition. Ecology 61: 358.

Goel N. and Lee T.M. 1995. Sex differences and effects of social cues on daily rhythms following phase advances in Octodon degus. Physiol. Behav. 58: 205.

- 1996. Relationship of circadian activity and social behaviors to reentrainment rates in diurnal Octodon degus (Rodentia). Physiol. Behav. 59: 817.

-1997. Social cues modulate free-running circadian activity rhythms in the diurnal rodent, Octodon degus. Am. J. Physiol. 273: R797.

Governale M.M. and Lee T.M. 2001. Olfactory cues accelerate reentrainment following phase shifts and entrain free-running rhythms in female Octodon degus (Rodentia). J. Biol. Rhythms 16: 489.

Gwinner E. 1966. Periodicity of a circadian rhythm in birds by species-specific song cycles (Aves, Fringillidae: Carduelis spinus, Serinus serinus). Experientia 22: 765.

Haim A. and Rozenfeld F.M. 1993. Temporal segregation in coexisting Acomys species: The role of odour. Physiol. Behav. 54: 1159.

Honrado G.I. and Mrosovsky N. 1989. Arousal by sexual stimuli accelerates the re-entrainment of hamsters to phase advanced light-dark cycles. Behav. Ecol. Sociobiol. 25: 57.

Huhman K.L. 2006. Social conflict models: Can they inform us about human psychopathology? Horm. Behav. 50: 640.

Jechura T.J., Mahoney M.M., Stimpson C.D., and Lee T.M. 2006. Odor-specific effects on reentrainment following phase advances in the diurnal rodent, Octodon degus. Am. J. Physiol. 291: R1808.

Kavaliers M. 1980. Social groupings and circadian activity of the killifish, Fundulus heteroclitus. Biol. Bull. 158: 66.

Levine J.D., Funes P., Dowse H.B., and Hall J.C. 2002. Resetting the circadian clock by social experience in Drosophila melanogaster. Science 298: 2010.

Marimuthu G., Rajan S., and Chandrashekaran M.K. 1981. Social entrainment of the circadian rhythm in the flight activity of the microchiropteran bat Hipposideros speoris. Behav. Ecol. Sociobiol. 8: 147.

Meerlo P., Sgoifo A., and Turek F.W. 2002. The effects of social defeat and other stressors on the expression of circadian rhythms. Stress 5: 15.

Menaker M. and Eskin A. 1966. Entrainment of circadian rhythms by sound in Passer domesticus. Science 154: 1579.

Mistlberger R.E. and Skene D.J. 2004. Social influences on mammalian circadian rhythms: Animal and human studies. Biol. Rev. Camb. Philos. Soc. 79: 533.

Mistlberger R.E., Antle M.C., Webb I.C., Jones M., Weinberg J., and Pollock M.S. 2003. Circadian clock resetting by arousal in Syrian hamsters: The role of stress and activity. Am. J. Physiol. 285: R917.

Morin L.P. and Cummings L.A. 1981. Effect of surgical or photoperiodic castration, testosterone replacement or pinealectomy on male hamster running rhythmicity. Physiol. Behav. 26: 825 .

Mrosovsky N. 1988. Phase response curves for social entrainment. J. Comp. Physiol. A 162: 35.

. 1996. Locomotor activity and non-photic influences on circadian clocks. Biol. Rev. Camb. Philos. Soc. 71: 343.

Potvin C.L. and Bovet J. 1975. Annual cycle of patterns of activity rhythms in beaver colonies (Castor canadensis). J. Comp. Physiol. 98: 243.

Rajaratnam S.M. and Redman J.R. 1999. Social contact synchronizes free-running activity rhythms of diurnal palm squirrels. Physiol. Behav. 66: 21.

Reebs S.G. 1989. Acoustical entrainment of circadian activity rhythms in house sparrows: Constant light is not necessary. Ethology 80: 172.

Refinetti R., Nelson D.E., and Menaker M. 1992. Social stimuli fail to act as entraining agents of circadian rhythms in the golden hamster. J. Comp. Physiol. A 170: 181. 


\section{CHRONOBIOLOGY OF COHABITATION}

Regal P.J. and Connolly M.S. 1980. Social influences on biological rhythms. Behaviour 72: 171.

Shemesh Y., Cohen M., and Bloch G. 2007. Natural plasticity in circadian rhythms is mediated by reorganization in the molecular clockwork in honeybees. FASEB J. 21: 2304.

Shkolnik A. 1971. Diurnal activity in a small desert rodent. Int. J. Biometeorol. 15: 115.

Weber E.T. and Hohn V.M. 2005. Circadian activity rhythms in the spiny mouse, Acomys cahirinus. Physiol. Behav. 86: 427.
Winfree A.T. 2001. The Geometry of biological time, 2nd edition. Springer-Verlag, New York.

Young L.J., Wang Z., Cooper T.T., and Albers H.E. 2000. Vasopressin $\left(\mathrm{V}_{1 \mathrm{a}}\right)$ receptor binding, mRNA expression and transcriptional regulation by androgen in the Syrian hamster brain. J. Neuroendocrinol. 12: 1179 .

Ziv Y., Abramsky Z., Kotler B.P., and Subach A. 1993. Interference competition and temporal and habitat partitioning in two gerbil species. Oikos 66: 237. 


\section{$8_{\mathrm{CSH}}^{\infty} \mathrm{C}$ Cold Spring Harbor Symposia SYMPOSIA on Quantitative Biology}

\section{On the Chronobiology of Cohabitation}

M. J. Paul and W. J. Schwartz

Cold Spring Harb Symp Quant Biol 2007 72: 615-621

Access the most recent version at doi:10.1101/sqb.2007.72.042

References This article cites 43 articles, 3 of which can be accessed free at:

http://symposium.cshlp.org/content/72/615.full.html\#ref-list-1

\section{License}

Email Alerting

Receive free email alerts when new articles cite this article - sign up in the box at the Service top right corner of the article or click here.

To subscribe to Cold Spring Harbor Symposia on Quantitative Biology go to: http://symposium.cshlp.org/subscriptions 